Pub. Mat. UAB

$N^{\circ} 21$ OCt. 1980

Actes VII JMHL

ALGUNOS ASPECTOS DE LA TEORIA DE CUBIERTAS RAMIFICADAS

Josë Ma Montesinos-Amilibia

Dpto. de Geometria y Topologia

Universidad de zaragoza

Trataremos de cubiertas de la 3 -esfera $s^{3}$ ramificadas sobre un enlace (= sistema de nudos), como un procedimiento de representar las 3-variedades cerradas y orientables.

La aplicación simplicial $\mathrm{f}: \mathrm{M}^{\mathrm{n}} \rightarrow \mathrm{N}^{\mathrm{n}}$ entre las $\mathrm{n}$-variedades compactas y trianguladas ${ }^{(1)} \mathrm{M}$ y $\mathrm{N}$ se llama cubierta ranificada si es una cubierta ordinaria fuera del (n-2)-esqueleto de $\mathrm{N}^{n}$. Los puntos de $\mathrm{N}^{n}$ cuya preimagen tiene menos puntos que hojas tiene la cubierta componen un subcomplejo $B^{n-2}$ llamado ramificación. Usaremos la locución "f es una cubierta de $\mathrm{N}^{\mathrm{n}}$ ramificada sobre $B^{\prime \prime}$.

Siendo este artículo de exposición, unicamente tocaremcs algunos temas selectos que ayuden a darse una idea de la teoria. El material es, en general, conocido, aunque algunos resultados son nuevos.

\title{
RAMIFICACION UNIVERSAL
}

En 1908 Tietze [T, p 104] pregunts, y en 1919 Alexander [A] probs que toda $\mathrm{M}^{\mathrm{n}}$ cerrada $\mathrm{y}$ orientable es una cubierta de $\mathrm{S}^{\mathrm{n}}$ ramificada. Ramirez [R] a petición de González-Acuña dio una version más fuerte de ese teorema:

(1) Siempre consideraremos variedades triangulares y conexas. 
Teorema 1 (Ramírez) Toda n-variedad cerrada y orientada es una cubierta de $\mathrm{s}^{n}$ ramificada sobre el (n-2)-esqueleto de un $n-s i m-$ ple:

Prueba del teorema 1.-

Se triangula $M^{n}$ y se dan orientaciones coherentes a los n-simples.

Se divide baricéntricamente la triangulación. Entonces cada nuevo n-simple, al tener un orden natural en sus vërtices, adquiere una orientación natural.

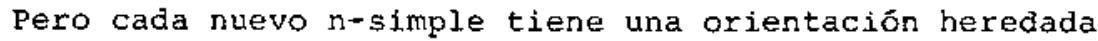
del n-simple viejo en el que descansa.

Un n-simple nuevo se colorea de negro si su orientación natural coincide con la heredada; en otro caso se colorea de blanco. El resultado es una coloración de la triangulacion en tablero de damas, pues n-simples nuevos, adyacentes a lo largo de una (n-1)-cara tienen orientaciones heredadas coherentes, pero orientaciones naturales incoherentes.

Sea $\mathrm{S}^{\mathrm{n}}$ formado pegando linealmente dos n-simples, uno negro $y$ otro blanco, por sus bordes, $y$ ordenense los ntl vertices resultantes.

La función $\mathrm{f}: \mathrm{M}^{\mathrm{n}} \rightarrow \mathrm{S}^{\mathrm{n}}$ envía un $\mathrm{n}-\mathrm{simple}$ blanco (negro) al $\mathrm{n}-$ simple blanco (negro) preservanto el orden de los vértices.

El siguiente corolario proporciona un nuevo modo de representar 3-variedades. Sea $G$ el subconjunto de $\mathrm{S}^{3}$ de Figura 1 .

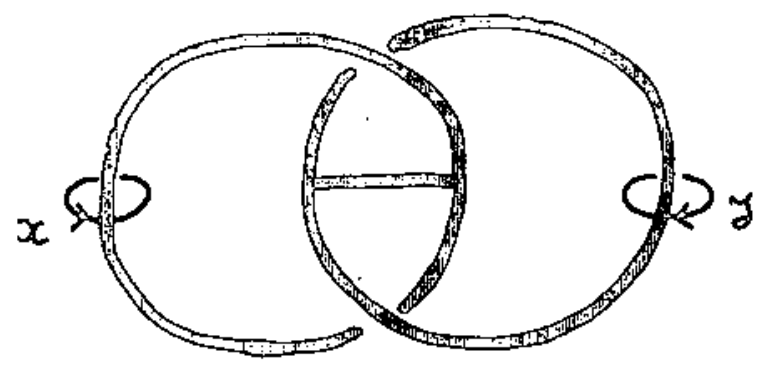

Figura 1 
Corolario 1. Toda 3-variedad cerrada y orientada es una cubierta de $\mathrm{s}^{3}$ con ramificación contenida en $\mathrm{G}$.

Prueba del corolario 1 . Por el teorema $1 \mathrm{M}^{3}$ es una cubierta de $\mathrm{s}^{3}$ ramificada sobre el 1 -esqueleto de un tetraedo $t$.

Supóngase $t$ contenido en $\mathbb{R}^{3} \subset \mathbb{R}^{3}+\infty$ y regular. Entonces una rotación de 120 e en torno a un eje de simetria trigonal de $t$, define una cubierta de $s^{3}$ sobre sí misma. La ramificación de esta cubierta junto con la imagen del l-esqueleto de $t$ es G.a

Es bien sabido (ver $\left[F_{1}\right]$, por ejemplo) que las cubiertas de $\mathrm{h}$ hojas de $\mathrm{S}^{3}$ ramificadas sobre $\mathrm{B}^{1}$ están en correspondencia biunivoca con las clases de equivalencia natural de representaciones transitivas de ${ }_{1}\left(S^{3}-B^{1}\right)$ en el grupo simetrico de grado $h$ $G_{h}^{\prime}$

Pero $\pi_{1}\left(s^{3}-G\right)$ es un grupo libre en los generadores $x$ e y de Fig. 1. Por tanto, dado un par $(\sigma, \tau)$ de permutaciones de $\sigma_{h}$. tendremos una representacion $w: \pi_{1}\left(S^{3}-G\right)+\sigma_{h}$ que sera transitiva si y s6lo si el subgrupo de $\sigma_{h}^{\prime}$ generado por $(\sigma, \tau)$ es transitivo. Por tanto podemos asociar al par transitivo $(\sigma, \tau)$ una pseudo-variedad $M(\sigma, \tau)$, a saber, la cubierta de $s^{3}$ ramificada sobre G asociada a la representación w. El corolario 1 se puede entonces anunciar asi:

Corolario 1'. Toda 3-variedad cerrada y orientada puede representarse como $\mathrm{M}(\sigma, \tau)$, para algun par transitivo de permutaciones $(\sigma, \tau)$.

La siguiente proposición facilita la confección de la "lista" de 3-variedades.

Proposicion 1. Se tienen las siguientes propiedades

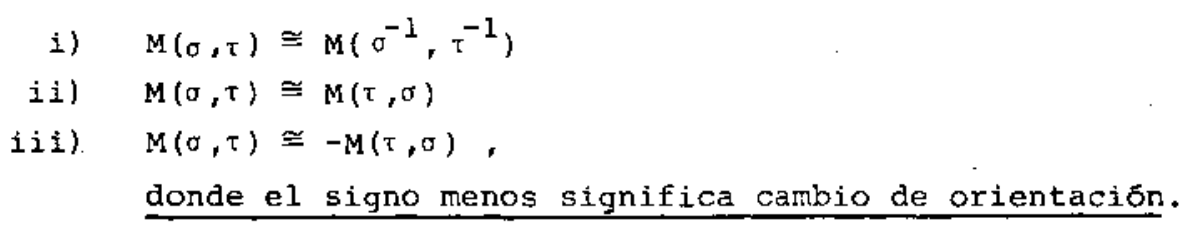




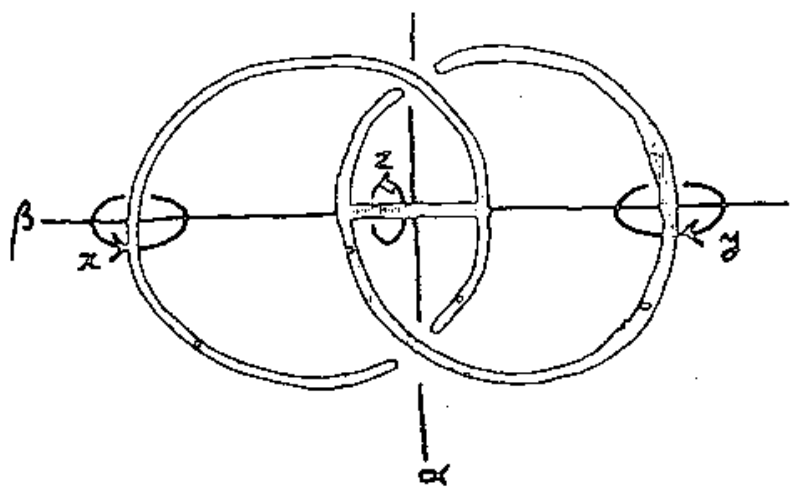

Figura 2

i) La simetría con respecto al eje B fija G como conjunto $y$ envia $(x, y)$ a $\left(x^{-1}, y^{-1}\right)$.

ii) La simetria con respecto al eje $\alpha$ fija $G$ como conjunto $y$ envia $(x, y)$ a $\left(y^{-1}, x^{-1}\right)$

iil) La simetria de $G$ con respecto al papel seguida de $1 a$ rotación de 180 o en torno a $B$ de uno de los circulos de $G$, fija $\mathrm{G}$ como conjunto $\mathrm{y}$ envia $(x, y)$ a $\left(x, y^{-1}\right)$ o

Corolario 2. $M(\sigma, \tau)$ es simëtrica si $\sigma=\sigma^{-1} \delta \tau=\tau^{-1} \cdot$.

Notese que $z=x y x^{-1} y^{-1}$ en ${ }^{\prime}\left(S^{3}-G\right)$, luego

Corolario 3. $M(\sigma, \tau)$ tiene (O) como ramificación si y sólo si $\sigma \tau=\tau \sigma$. En este caso la cubierta es regular. $\square$

Ejemplos: 1.- Si $\sigma=1$, entonces $t=(12 \ldots h)$ y la cubierta $M(1, \tau)=s^{3}$

2.- Si $\sigma=\tau$, entonces $0=\tau=(12 \ldots h)$ y la cubierta es crclica de h hojas ramificada sobre $D$, y la cubierta $M(\sigma, \sigma)$. es la lente $L(h, 1)$. Por tanto $M\left(\sigma, \sigma^{-1}\right)=-L(h, 1)$. Notese que $a=(123) \neq(132)=\sigma^{-1}, y$ en efecto $L(3,1) \neq-L(3,1)[S T$.

3.- Damos sin demostracion algunos ejemplos: $\left.M(12,13)=s^{3}, M(12)(34),(13)(24)\right)=s^{3}, M(12,123)=s^{1} \times s^{2}$.

Como se ve, la anterior representacion, cuyo estudio abandonamos aqui tras esas breves observaciones, tiene la importancia de proporcionar una ramificacion universal. Pero notese que el no de hojas puede ser arbitrariamente alto (1).

(1) Puede verse esto considerando $M^{3}=h * s^{1} \times s^{2}$. Aqui $\pi_{1} M^{3}$ es libre con $h$ generadores $y$ h tan alto como queramos. 


\section{MINIMO NUMERO DE HOJASS}

Se trata ahora de averiguar si toda $M^{n}$ puede representarse como cubierta de $S^{n}$ ramificada, de modo que el no de hojas se mantenga bajo cierta cota.

Como entonces la ramificación dejara de ser universal, desearemos que se mantenga dentro de una clase de subcomplejos de $S^{n}$ manejable. Por ejemplo, ces toda $M^{n}$ cubierta de $S^{n}$ ramificada sobre una subvariedad de $\mathrm{s}^{\mathrm{n}}$ ?. La respuesta es negativa si se exige que la subvariedad sea localmente plana [EB]:

Teorema 2 (Edmonds y Berstein). Sea $M^{n}$ una variedad diferenciable spin, que és cubierta de $\mathrm{S}^{\mathrm{n}}$ ramificada sobre una subvariedad localmente plana. Entonces todos los productos de clases de Stiefel-Whitney de $M^{n}$ valen cero.

Nota.- Esto implica que todos los numeros de Stiefel-Whitney, excepto por el fltimo que es la caracterfstica de Euler módulo 2 , valen cero. Luego si adems de las condiciones del teorema, $M$ tiene caracteristica de Euler par, se deduce que $M^{n}$ es borde de alguna $Q^{\text {ntl }}$ (Teorema de Thom).

Por ejemplo el plano proyectivo cuaterniónico $Q \mathrm{P}^{2}$ es spin ya que tiene cohomologra sólo en dimensiones 0,4 y 8 . Como $\mathrm{w}_{4}^{2} \neq 0, Q \mathrm{P}^{2}$ no puede cubrir $\mathrm{s}^{8}$ con ramificación una 6-variedad localmente plana [EB] .

También para el nümero minimo de hojas se conocen restricciones [E].

Teorema 3 (Edmonds). Supongase que $M^{\mathrm{n}}$ es cubierta ramificada de $s^{n}$ con $h$ hojas. Entonces el maximo numero de clases de cohomologia reducida entera de $\mathrm{M}$ cuyo producto no es cero, no puede rebasar h.

Por ejemplo, este numero es $n$ para el $n$-toro $T^{n}$. Luego, si $\mathrm{T}^{\mathrm{n}}$ es cubierta ramificada de $\mathrm{s}^{\mathrm{n}}$, el no de hojas es $\geq \mathrm{n}$. Para $\mathrm{T}^{3}$ esto fue demostrado primero por $\operatorname{Fox}\left[\mathrm{F}_{2}\right]$. 
Pasando ahora a resultados positivos, vemos que toda_2-variedad cerrada $y$ orientable es una cubierta de dos hojas de $s^{2}$ ramificada (examinese la Fiqura 3 ).

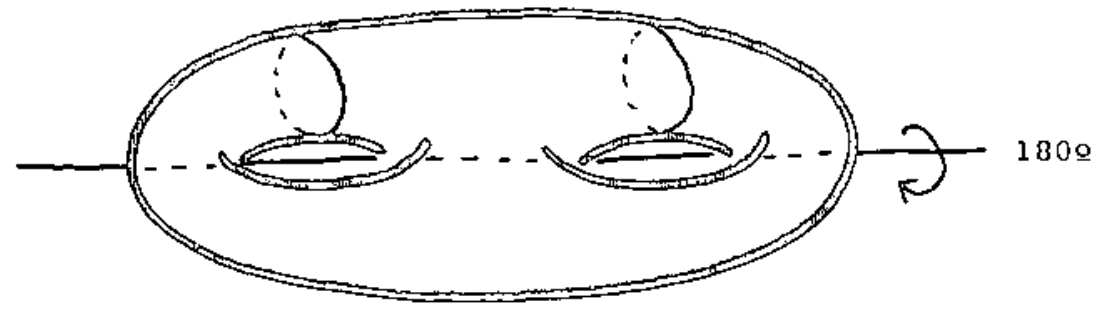

Figura 3

Para $n=3$ tenemos el. siguiente resultado obtenido independientemente por Hilden [H] $y$ el autor $\left[M_{1}\right]$.

Teorema 4 (Hilden y Montesinos). - Toda. 3-variedad cerrada y orientada es una cubierta, diedral de tres hojas, de $s^{3}$ ramificada sobre un nudo.

Nota. "Diedral" significa que el grupo diedral $6_{3}$, de seis elementos, es el gxupo de monodromía. Así la variedad corresponde a una cierta representación transitiva $\omega: \pi{ }_{1}\left(S^{3}-K\right) \rightarrow \sigma_{3} \rightarrow 0$, en donde $K$ es un nudo. Por tanto w representa necesariamente meridianos de $\mathrm{K}$ por trasposiciones. Luego la cubierta es irregular pues el estabilizador de un indice no es normal. Ass un punto de $\mathrm{K}$ tiene exactamente dos preimágenes, de índices de ramificación dos $y$ uno.

Esbozo de 1a prueba del teorema 4.- Seguiremos la nueva prueba de este teorema publicado en $\left[\mathrm{x}_{2}\right]$.

El punto de partida es la representacion de 3 -variedades, debida a Iickorish [L] y wallace [W], mediante cirugra en un enlace de $\mathrm{s}^{3}$. 
Notamos que $\mathrm{s}^{3}$ satisface el teorema, pues la cubierta diedral de $\mathrm{s}^{3}$ ramificada sobre el enlace trivial de dos componentes $L$, asociada a la cinica posible representacion de $\pi_{1}\left(S^{3}-L\right)$ sobre $G_{3}$, es $S^{3}$. Llamemos $P Y Q$ a las componentes de $L y$ sean $P_{12}, P_{3}$ (resp. $Q_{13}, Q_{2}$ ) las preimágenes de $P$ (resp. $Q$ ) de indices de ramificación dos $\mathrm{y}$ uno. As $\mathrm{P}_{12}, \mathrm{P}_{3}, \mathrm{Q}_{13}, \mathrm{Q}_{2}$ es un enlace trivial de cuatro componentes y la cubierta $f: s^{3} \rightarrow s^{3}$ pliega $s^{3}$ sobre si mismo con lineas de plegado $P_{12}$ y $Q_{13}$, del modo en que se hacen tres dobles en una carta.

Supongamos para facilitar que la variedad problema viene dada por cirugía en el nudo $\mathrm{N}$ de la figura 4.

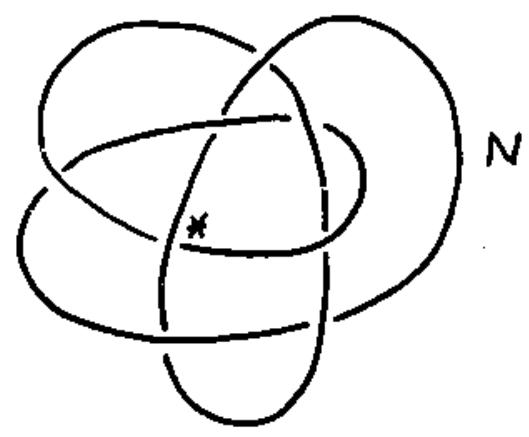

Figura 4

Entonces "simetrizamos" el nudo con respecto a los dos ejes $P_{12}, Q_{13}$. Esto se realiza en dos pasos. Primero cambiamos $\mathrm{N}$ en $\mathrm{N}^{\prime}$. para hacerlo simétrico con respecto a $\mathrm{P}_{12}$ (Figura 5). 

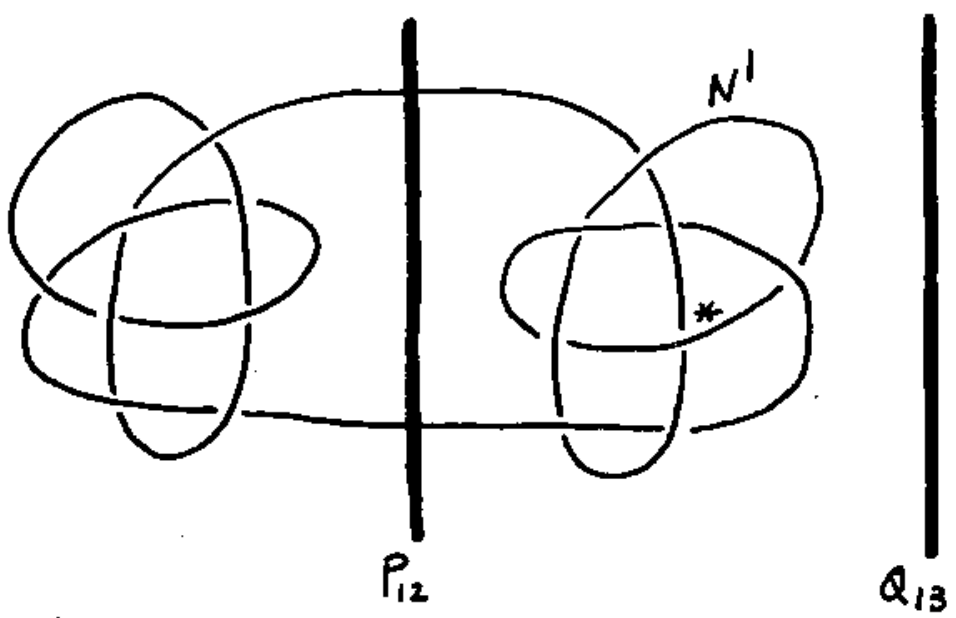

Figura 5

Después localizamos puntos dobles de la proyección de $\mathrm{N}$ en los que un cambio local de pasos superiores en inferiores convierte $\mathrm{N}$ en el nudo trivial (en el nudo $\mathrm{N}$ basta el punto indicado en Figura $\dot{4} j$.

Introducimos, en torno al correspondiente punto doble de $N^{\prime}$ (Figura 5), una componente trivial $T$, que puede hacerse simetrica con respecto a T. (Figura 6).

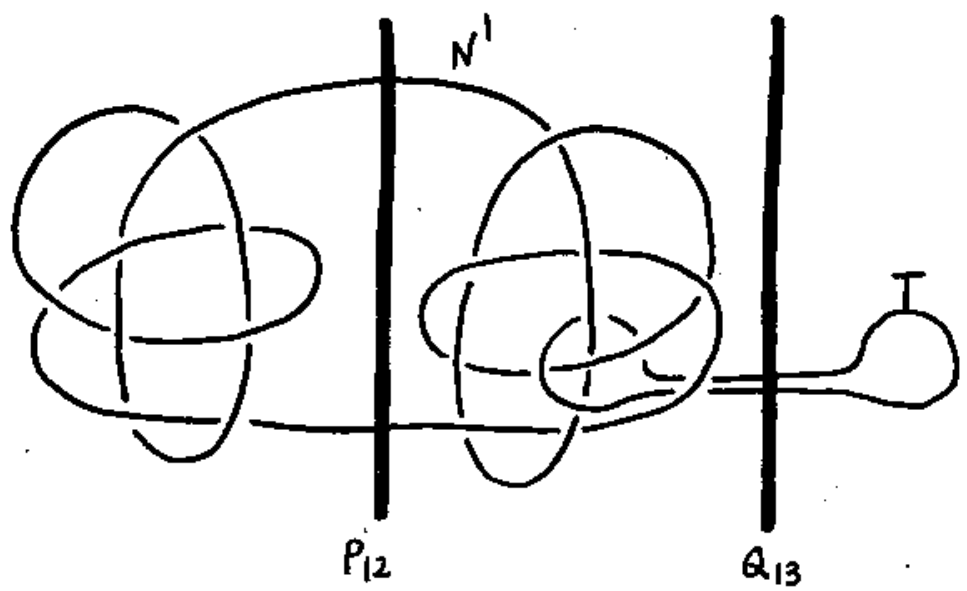

Figura 6

116 
La conversión de $\mathrm{N}$ en $\mathrm{N}$ ' U $\mathrm{T}$ es lo que hemos llamado simetrización más arriba. Pues bies, $\mathrm{M}^{3}$ se obtiene por cirugía en N'UT. En efecto, aplicando el truco de hempel [ H] a $\mathrm{T}$ se produce un cambio local de un paso superior en inferior y una conversion de $\mathrm{N}^{\prime}$ en $\mathrm{N}$.

La imagen de $N^{\prime} \cup T$ mediante f está formada de dos arcos cuyos extremos descansan en L (Figura 7)

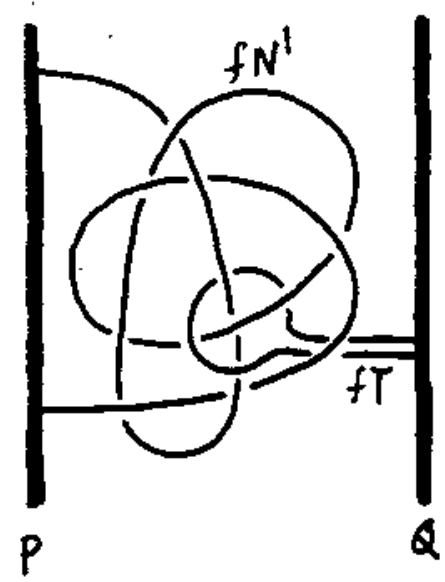

Figura 7

Resta convencerse de que cualquier cirugfa realizada en N'U T es compatible con el pliege que define $f$. Pero notese que este pliege restringido a $N^{\prime} \sigma$ a $T$ viene generado por una simetría axial. Luego cualquier curva simple que descanse en la frontera de un entorno regular de $N^{\prime} \delta \mathrm{T}$ es simétrica con respecto a tal simetría. Interpretando tal curva como el nuevo meridiano de la cirugía, vemos que esta se proyecta por $f$, produciendo una modificaciốn en el entorno de $\mathrm{N}^{\prime} 6 \mathrm{~T}$, que no altera el tipo topológico de $s^{3}$ (truco de Alexander).

La prueba se acabará cuando consigamos una ramificación con una sóla componente. Esto lo veremos en la siguiente sección.

El teorema anterior es constructivo, pero, en la práctica, el proceso de simetrización puede acortarse, como en los dos. ejemplos siguientes.

Ejemplo. El nudo de la figura 4 se puede simetrizar, economizando puntos dobles, como en la figura 8 . 


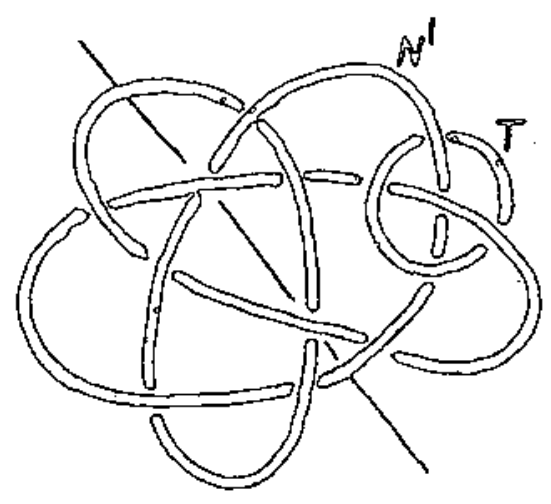

\section{Figura 8}

Ejemplo (Anillos de Borromeo) El enlace de la figura 9a es simétrico (Figura $9 b$ ).

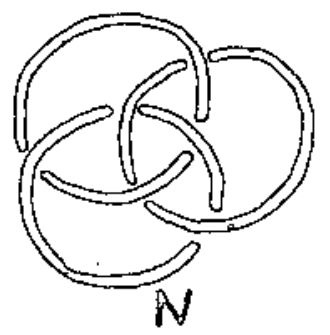

9 a

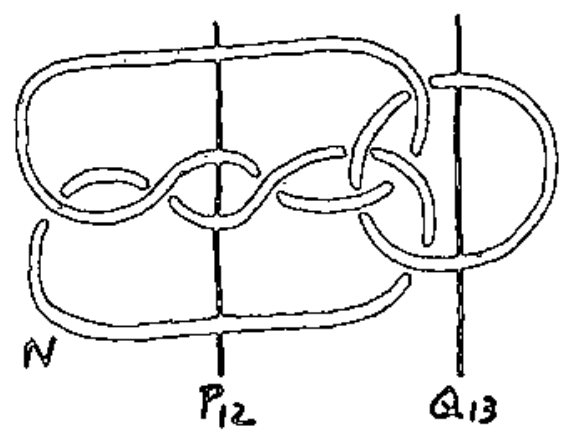

$9 \mathrm{~b}$

Figura 9 
El toro tridimensional $\mathrm{T}^{3}$ se obtiene por 0-cirugía en los anillos de Borromeo. La ramificación de la cubierta $\mathrm{T}^{3} \rightarrow \mathrm{S}^{3}$ dada por el teorema 4 (y figura 9 b) aparece en Figura 10

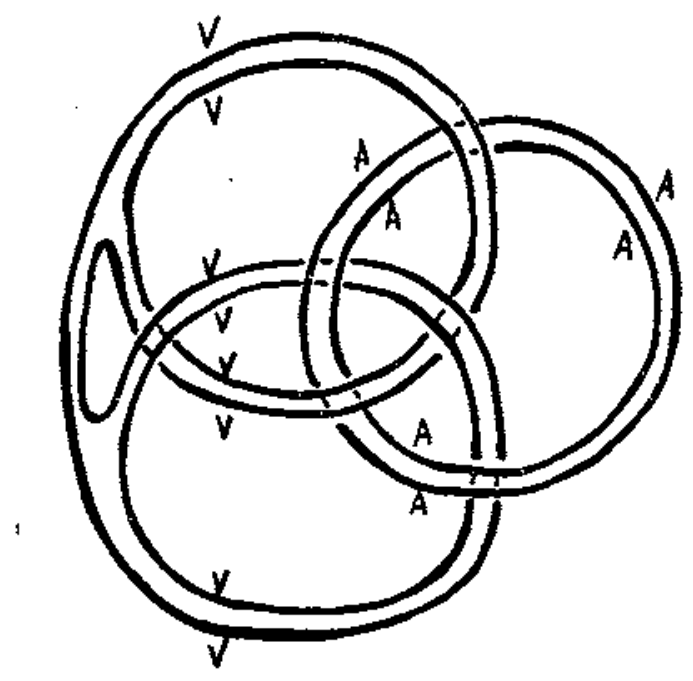

Figura 10

El anterior teorema no puede mejorarse exigiendo, por ejemplo, que la cubierta sea regular. En efecto, Tollefson [T] ha hallado ejemplos de 3-variedades que no admiten automorfismos periodicos; sus cubiertas ramificadas sobre $\mathrm{s}^{3}$ tienen entonces grupo trivial de superposiciones, $y$, asf, no pueden ser regulares.

\section{ENLACES COLOREADOS}

Un modo sugestivo de dar un enlace $L$ junto con una representación $\omega: \pi_{1}\left(S^{3}-E\right)+G_{3}$, ideado por Fox, consiste en colorear los pasos del enlace $L$ usando tres colores (V=verde, $A=a z u I$ y $R=r o j o$, por ejemplo) $(1)$ de modo que los tres colores de junto

(1) Sugerimos que el lector ilumine por sî mismo las figuras. 
a un cruce sean o todos iguales o todos distintos; pues basta hacer $V=(12), A=(13), R=(23)$ y asignar a cada meridiano de I su color (o permutación) para tener w. La representación es transitiva si se usan los tres colores (el enlace de Figura 10 está coloreado de modo que la cubierta asociada es $\mathrm{T}^{3}$ ).

podemos entonces enunciar el Teorema 4 as

Teorema $4^{\prime}$. Toda 3-variedad cerrada y orientable está representada por un nudo coloreado.

Conclusión de la prueba del teorema $4^{\prime}$ - Hemos visto que toda 3-variedad viene dada por un enlace coloreado. para acabar la prueba consideramos ciertas modificaciones de un nudo coloreado introducidos en $\left\{_{3}\right\}$.

Lema 1 (Montesinos) La jugada
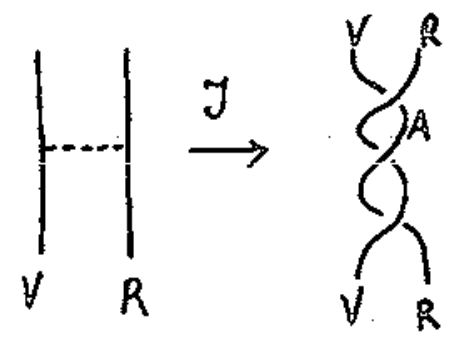

aplicada a un enlace coloreado no cambia la cubierta de $\mathrm{s}^{3}$ asociada

Esbozo de prueba del lema l. El arco de puntos de la figura se levanta a un arco. Ia jugada $\mathcal{H}$ se levanta a una modificación en el entorno de un arco y esto no cambia la cubierta.

Notese ahora gue $Y$ permuta los dos ramales. ApIicando varias veces a un enlace coloreado resultara un nudo coloreado.

La jugada $y$ sugiere un interesante

Problema 1. Hallar una ststema de jugadas de un enlace coloreado que no alteran la cubierta asociada y tales que dos nudos coloreados de igual cubierta estan relacionados por una sucesión

finita de jugadas. 
EI interés de este problema es que si tiene respuesta positiva, dispondremos de una traducción, en términos de nudos coloreados, del problema de clasificación de 3-variedades cerradas $y$ orientadas.

Observese que en el entorno del arco punteado del Lema $I$, pueden hacerse modificaciones más complicadas que $\mathcal{Y}$ y que tambien se levantan. Pero todas ellas dependen de $Y$. En efecto las modificaciones más generales aparecen en figura 11
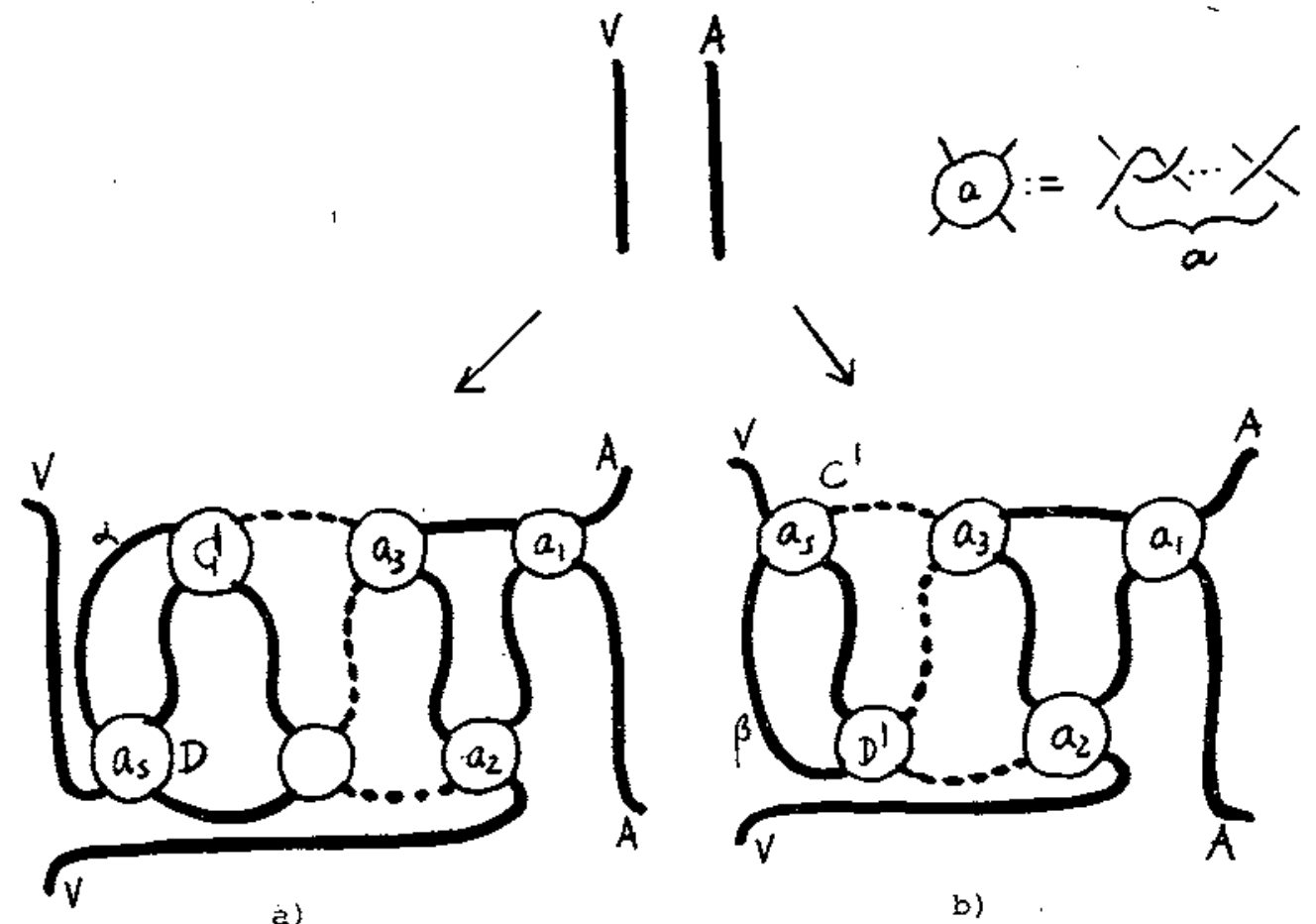

ᄅ)
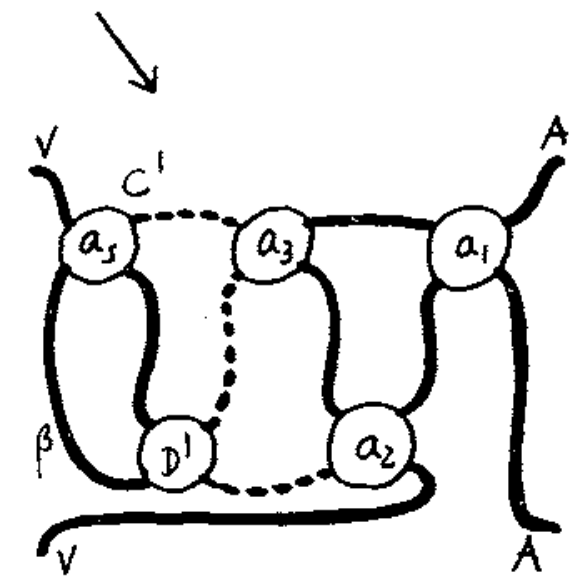

b)

Figura 11 
Pero a no puede ser verde, luego tras aplicar jugadas $y$ el ovizlo $D$ tendra cero $\delta \pm j$ cruces. Si tiene cero cruces podemos eliminar los ovillos C y D. Si tiene $\pm I$ cruces podenos incorporar D al ovillo C obteniendo una situación como la de la Fig. I Ib). Pero $\beta$ no es verde, luego tras aplicar jugadas $y$ el ovilio $C^{\prime}$ tendrá cero ó \pm 1 cruces, etc... Despuss de un nGmero finito de pasos no quedará ningun ovillo. Asi las jugadas de la Fig. Il dependen de $Y_{-}$.

En principio esto no significa que la jugada $y$ baste para resolver el anterior problema. Son, en efecto, concebibles otras jugadas.

Los dos nudos coloreados de la Figura 12 tienen igual cubierta asociada $1 \mathrm{M}_{4} \mathrm{l}$. Estân relacionados entre si mediante jugadas
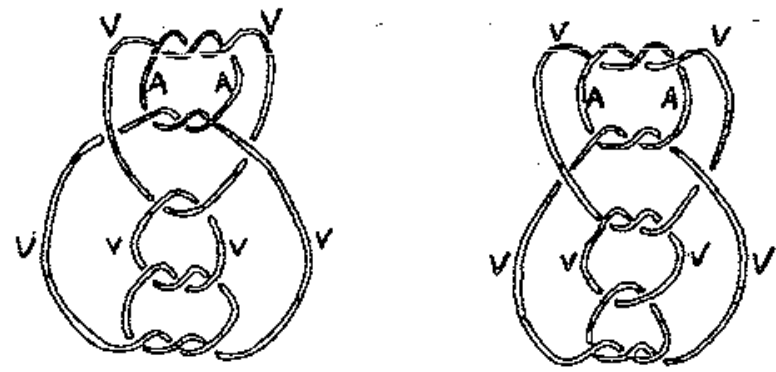

Figura 12 
Obsërvese como los nudos coloreados de Figura 13 se "separan" mediante aplicación de jugadas $y$ en los lugares marcados.
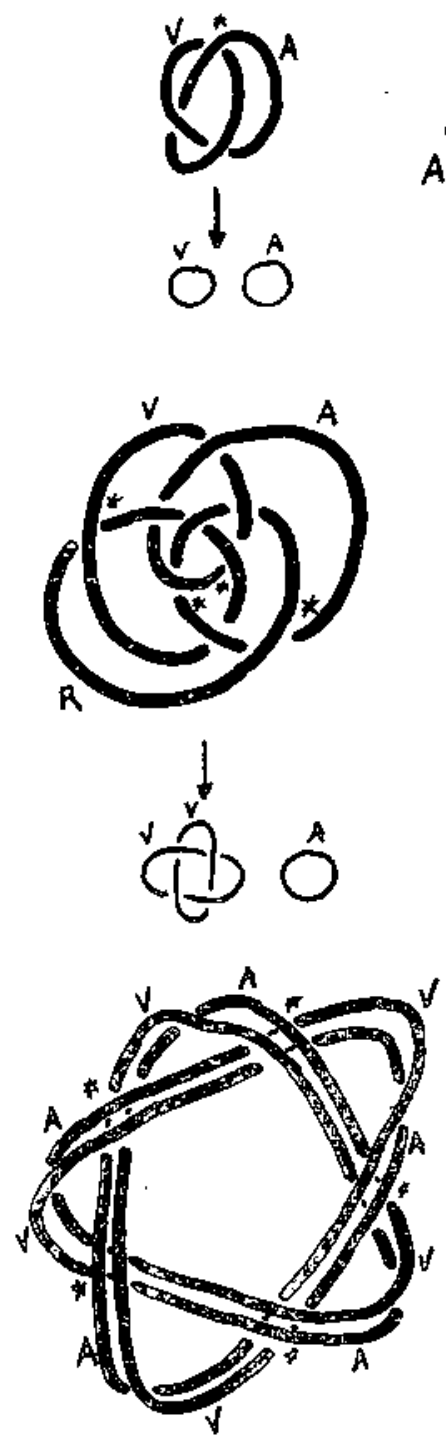
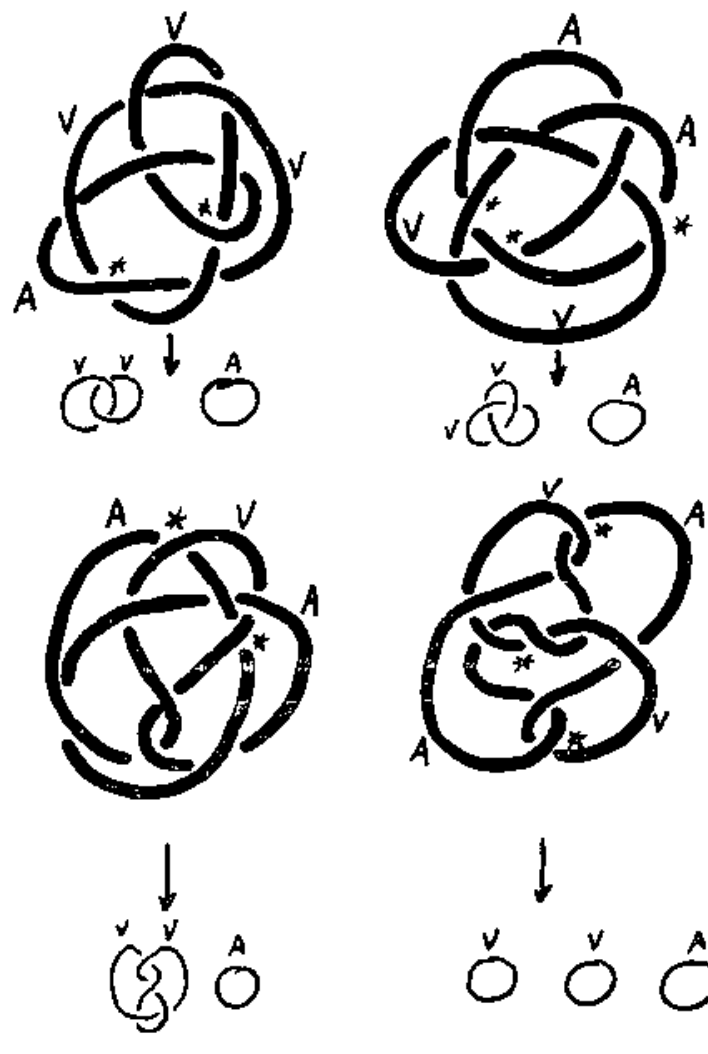
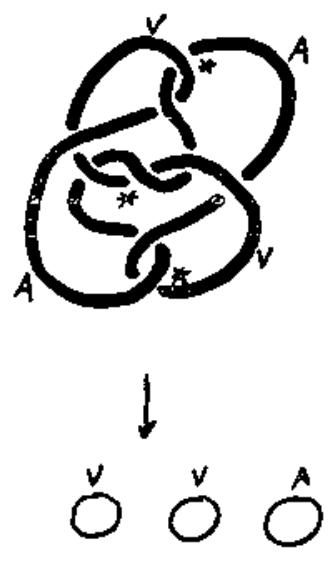

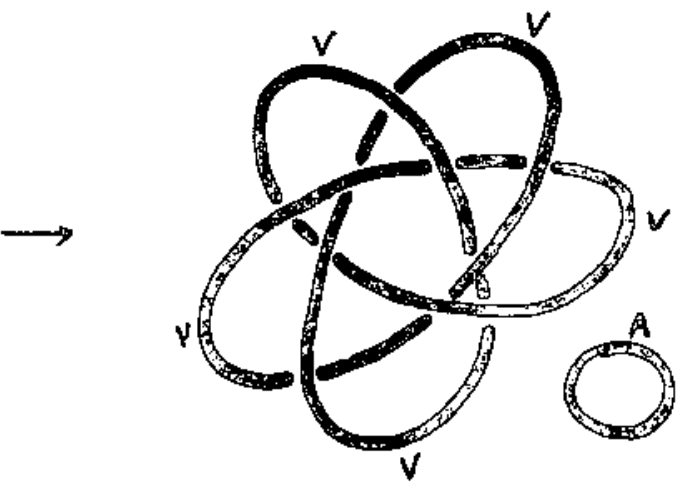

Figura 13

(1) El material de esta seccion esta tomado de $\left[M_{3}\right]$ (ver también $\left[F_{2}\right]$. 
Diremos que un enlace coloreado es separable si mediante jugadas $y$ se convierte en un enlace separado $L_{1} \cup L_{2}$ en el que $L_{1}$ esta coloreado de verde $\mathrm{y}_{2}$ de azul

El lector puede ejercitarse viendo que toda trenza de 2 - 3 filamentos coloreada, es separable $\left[\mathrm{M}_{3}\right]$. Sin embargo, hay algunos nudos coloreados no separables, como se sacara del siguiente teorema.

Teorema 5. La variedad asociada a un enlace separable es una cubierta ciclica de dos hojas de $\mathrm{s}^{3}$ ramificada sobre una suma de $\mathrm{L}_{1} \mathrm{Y} \mathrm{L}_{2}$.

Esbozo de la prueba del teorema 5.

Una 2-esfera que separa $L_{1}$ de $L_{2}$ se levanta a una 2-esfera gue define la suma conexa de $\widetilde{L}_{1}, \widetilde{L}_{2}$ (cubiertas dobles ramificadas

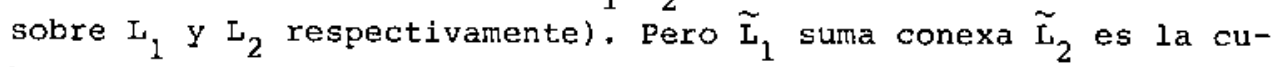
bierta doble de una suma de $L_{1} y L_{2}$, como se ve levantando una 2-esfera que defina dicha suma.

Las variedades asociadas a los ejemplos de Figura 13 son todas cubiertas dobles de $s^{3}$; a saber (y por orden) $s^{3}, \mathbb{R} \mathrm{P}^{3}$, $L(3,1), L(4,1), L(5,2), s^{1} \times s^{2}$ y esfera homol6gica de poincaré (cubierta doble del toroidal $(3,5)$ [sw]).

El teorema 5 junto con el teorema 3 implican que hay nudos coloreados no separables. Un ejemplo es el enlace coloreado de Figura 10, pues su variedad asociada es $\mathrm{T}^{3}$ que no es cubierta doble de $s^{3}$ (el primer ejemplo de enlace no separable se debe a Fox $\left[\mathrm{F}_{2}\right]$.).

Problema 2. Un nudo coloreado cuya cubierta asociada es cubierta doble ramificada de $\mathrm{s}^{3}$ ces separable?

Problemas $\lambda$ y 2 estan relacionados.

Conjetura 1 (Fox $\left[F_{2}\right]$ ) Un nudo coloreado cuya cubierta asociada es simplemente conexa, es separable

La conjetura l implica la conjetura de Poincaré. Pues si e.t nudo es separable, la variedad es cubierta doble ramificada de $\mathrm{s}^{3}$ y para estas la conjetura de Poincare es valida (1).

(1) Este reciente resultado es consecuencia de la llamada "Conjetura de Smith fuerte". Consfiltense las actas isin publicar todavia) del simposio habido sobre este tema en la universidad de Columbia. 
La afirmación de que la conjetura de Poincare implica la conjetura 1 , es la solucion del problema 2 en el caso de gue 1 a cubierta sea $\mathrm{s}^{3}$.

\section{REFERENCIAS}

R A. RAMIREZ: "Sobre un teorema de Alexander" Anales del Instituto de Matematicas de la UNAM 15(1975) 77-81.

T H.TIETZE: "Uber die topologischen Tnvarianten mehrdimensionaler Mannigfaltigkeiten" Monatsh.Math. 19(1908) $1-118$.

A J.W.ALEXANDER: "A note on Riemann spaces" Bull. Amer.Math. Soc. 26 (1919) $370-372$.

ST H.SEIFERT y w.THRELFALL: "Lehrbuch der Topology" Leipzig und Berlin, 1934.

F. R.H.FOX: "A,quick trip through knot theory" Topology of 3-manifolds and related topics, Englewood Cliffs (1962) $120-167$.

E A.L.EDMONDS: "The degree of a branched covering of a sphere" Geometric Topology, Academic Press (1979), 337-343

BE I.BERSTEIN y A.L.EDMONDS: "The degree and branch set of a branched covering" Invent. Math.

$M_{1}$ J.M.MONTESINOS: "A representation of closed, orientable 3-manifolds as 3-fold branched coveringsof $\mathrm{s}^{3}$ " Bull. Amer.Math.Soc. 80 (1974) 845-846

H H.M.HIEDEN: "Every closed, orientable 3-manifold is a 3-fold branched covering space of $\mathrm{s}^{3}$ " Bull. Amer.Math. Soc. 80 (1974) 1243-1244.

$F_{2}$ R.H.FOX: "A note on branched cyclic coverings of spheres" Rev.Mat.Hisp. Amer. $32(1972)$ 158-166.

L W.B.R.LICKORISH: "A representation of orientable combinatorial 3-manifolds" Annals of Math. 76(1962) 531-540

W A.D.WALLACE: "Modifications and cobounding manifolds" Cand. J.Math. (1960) 503-528

$\mathrm{M}_{2}$ J.M.MONTESINOS: "A note on 3-fold branched covering of $\mathrm{s}^{3}$ " Math.Proc.Camb.Phi. Soc. (1980)

SW C.WEBER y H.SEIFERT: "Die beiden Dodekaederräume" Math. 2 . $37(1933) 237-253$. 
$M_{3}$ J.M.MONTESINos: "Sobre la conjetura de Poincaré y los recubridores ramificados sobre un nudo". Tesis doctoral, Madrid (1971).

H J.HEMPEL: "Construction of orientable 3-manifolds" Topology of 3-manifolds and related topics. Englewood cliffs (1962) 207-212.

T J.L.TOLLEFSON: "A 3-manifold with no PL involutions" Notices of the Amer.Math.Soc. $22(19 / 5)$ A-231.

$M_{4}$ J.M.MONTESINOS: "Variedades de seifert que son recubridores cíclicos ramificados de dos hojas" Bol.Soc.Mat. Mex. 18 (1973) 1-32. 